\title{
PERFIL GENERAL DE LAS USUARIAS DEL PROGRAMA DE INTERRUPCIÓN DEL EMBARAZO EN UNA UNIDAD DE SALUD REPRODUCTIVA DE LA CIUDAD DE MÉXICO
}

\author{
María de Lourdes Jacob-Araiza ${ }^{1}$ E Efrén Raúl Ponce-Rosas ${ }^{2 *}$ \\ 'Especialista en Medicina Familiar, Secretaría de Salud; ${ }^{2}$ Especialista en Medicina Familiar, profesor de la División de Estudios de Posgrado, \\ Facultad de Medicina, UNAM. México
}

RESUMEN: Objetivo: Describir el perfil general de las mujeres usuarias del Programa de Interrupción Legal del Embarazo en una unidad de salud reproductiva de la Ciudad de México. Diseño: Estudio descriptivo, transversal y retrospectivo. Material y métodos: Un total de 7,177 registros del Servicio de Estadística (20122014) del Programa de Interrupción Legal del Embarazo, los cuales se realizaron en una unidad de salud reproductiva de la Ciudad de México. Intervención: Se recolectaron variables sociodemográficas y clínicas, y se registraron en el formato oficial administrativo del servicio de estadística. Análisis: Descriptivo e inferencial, (chi cuadrada, valor $\mathrm{p}<.05$ ), SPPS versión 22 . Resultados: Se revisaron los registros de 7,117 interupciones legales del embarazo (ILE). El grupo de edad con mayor frecuencia fue el de 21 a 25 años (34\%). El 76\% de las mujeres tenían un nivel de escolaridad de secundaria y preparatoria. Respecto al estado civil, el $54 \%$ eran solteras y el $26 \%$ estaban en unión libre. Al total de las mujeres que se les realizó la interrupción se les ofreció y otorgó algún método de anticoncepción de acuerdo a las normas según su edad, condiciones clínicas y elección de las pacientes; los métodos hormonales fueron los más frecuentes, con un $75 \%$ (implante subdérmico, parches, orales e inyectables). Se encontraron diferencias significativas en la edad, estado civil y métodos de anticoncepción $(\mathrm{p}<0.001)$. Conclusiones: Se contribuye a la generación de información general útil para la pla-
GENERAL PROFILE OF THE USERS OF THE PREGNANCY INTERRUPTION PROGRAM IN A REPRODUCTIVE HEALTH UNIT IN MEXICO CITY

ABSTRACT: Objective: Describe the general profile of women using a Legal Pregnancy Interruption Program in a reproductive health unit in Mexico City. Design: Descriptive, cross-sectional, retrospective. Material and methods: A total of 7,177 records from the statistical service (2012-2014) of the Legal Pregnancy Interruption (LIP) Program in a reproductive health unit in Mexico City were analyzed. Intervention: Sociodemographic and clinical variables were collected and registered in the official administrative form of the statistics service. Analysis: Data were analyzed in SPSS v 22 with descriptive and inferential statistics (Chi square with a p value <.05). Results: A total of 7,117 legal pregnancy interruption records were reviewed. The most frequent age group was 21 to 25 years with $34 \%$. Seventy six percent of women had a secondary and preparatory school level; regarding marital status, 54\% were single and $26 \%$ in common law marriage. All of the women who had an interruption were offered and granted some method of contraception according to the regulations, their age, clinical conditions, and the patient's choice. Hormone methods were more frequent in $75 \%$ (subcutaneous implant, patch, oral and injectable). Significant differences were found in age, marital status and contraceptive methods $(\mathrm{p}<0.001)$. Conclusions: The use of the Legal Interruption of Pregnancy Program is greater in single women 21 to 25 years of age with a secondary and preparatory education.
Correspondencia:

*Efrén Raúl Ponce-Rosas

E-mail:ipr@unam.mx
Fecha de recepción: 06-04-2018

Fecha de aceptación: 15-05-2018

doi:10.24875/RMF.18000078 
neación, diseño y orientación de las políticas de atención y mercadotecnia en salud de las mujeres que solicitan ILE.

Palabras clave: Interrupción del embarazo. Atención primaria. Métodos de anticoncepción. Aborto.
This contributes to the generation of useful general information for the planning, design and orientation of health care and marketing policies for women users. (Rev Mex Med Fam. 2018;5:68-74) Corresponding author: Efré Raúl Ponce-Rosas, ipr@unam.mx

Key words: Pregnancy interruption. Primary care. Contraception methods. Abortion.

\section{INTRODUCCIÓN}

En las guías de práctica clínica de la Secretaría de Salud de México se define aborto como la terminación espontánea o provocada de la gestación antes de la vigésima semana, contando desde el primer día de la última menstruación normal o expulsión de producto de la gestación con un peso menor a $500 \mathrm{~g}^{1}$.

A pesar de los avances de salud, de nuevas tecnologías y logros en los derechos humanos para otorgar una atención segura e integral para la realización de abortos, se estima que cada año se realizan 22 millones de abortos en el mundo de manera insegura, el 98\% de los cuales se llevan a cabo en países en vías de desarrollo, cantidad que aumentó de 20 millones en 2003 a 22 millones en $2008^{2}$. Este fenómeno causa la muerte de alrededor de 47,000 mujeres y discapacidades en otros 5 millones como resultado de las complicaciones de un aborto inseguro. Muchas de estas muertes podrían haberse evitado si se lograra el acceso a una educación sexual, planificación familiar y al aborto inducido de forma legal y sin riesgo ${ }^{2}$.

En el año 2012, el Centro de Noticias de la Organización de Naciones Unidas mencionó que, en un estudio publicado en la revista médica Lancet, en América Latina el 95\% de los abortos eran inseguros, proporción que no cambió entre 1995 y 2005, y agregó que casi todos los procedimientos seguros se realizaron en Cuba, donde el aborto es legal y las mujeres pueden optar por él con libertad ${ }^{3}$.
Para el año 2009, según un reporte de Salud Pública, la interrupción de embarazos no deseados en condiciones inadecuadas constituyó el 13\% de mortalidad materna en todo el mundo ${ }^{4}$. Es difícil cuantificar el número de abortos en la República Mexicana, ya que la mayor parte se hace en la clandestinidad y no se cuenta con cifras confiables. En una investigación publicada en 2012, se estimó que cada año se practican 53,000 abortos. En México el aborto es un problema social y de salud pública, pues cada 45 días mueren ocho mujeres por abortos mal practicados, esto es, un deceso cada nueve días. La mayoría son mujeres pobres y jóvenes ${ }^{5}$.

En América Latina y el Caribe se realizan cada año alrededor de 4 millones de abortos inseguros $^{6}$. En la Ciudad de México, la interrupción del embarazo por la sola voluntad de la mujer es legal en las primeras 12 semanas de gestación. El 24 de abril de 2007 la Ley en el Distrito Federal (hoy Ciudad de México) aprobó la reforma del código penal, art. 144 al 147, en los hospitales del Gobierno del Distrito Federal ${ }^{4}$.

La Alianza Nacional por el Derecho a Decidir (ANDAR) surgió en 2002 como parte del movimiento de mujeres en México, es el espacio que vincula a diversas organizaciones y personas, principalmente de los sectores de la salud, derecho y educación, con el propósito de fortalecer el ejercicio de los derechos sexuales y reproductivos de todas las personas en México. Tres de las principales agrupaciones de ANDAR son: International 
Projects Assistance Services (Ipas) México, Population council y Grupo de Información en Reproducción Elegida (GIRE) ${ }^{7}$.

El GIRE reporta que en la Ciudad de México se concentraron el 14\% del total de muertes por aborto, y ésta es considerada como la tercera causa de muerte materna. Este porcentaje cambió con el Programa de Interrupción Legal del Embarazo hasta las 12 semanas de gestación ${ }^{5}$.

El objetivo de este estudio fue describir el perfil general de las mujeres usuarias que solicitaron ILE en una unidad de salud de la Ciudad de México.

\section{MATERIALY MÉTODOS}

Diseño: descriptivo, transversal y retrospectivo.

Población, lugar y tiempo: 7,177 registros oficiales del Servicio de Estadística (2012-2014) de mujeres atendidas en el Programa de Interrupción Legal del Embarazo que se realizaron en una unidad de salud reproductiva de la Ciudad de México. La población estudiada fue el total de los registros administrativos oficiales, correspondientes a igual número de procedimientos de ILE, los cuales se realizaron en el periodo comprendido entre 2012 y 2014. Se obtuvo información de variables sociodemográficas y clínicas. Los datos fueron medidos en una sola ocasión y registrados en el formato oficial administrativo del Servicio de Estadística perteneciente a la unidad de salud. El acceso a la información fue autorizado por el director de la unidad de salud. Además, se garantizó el anonimato y las mujeres que se realizaron la ILE firmaron previamente el consentimiento informado legal para practicar el procedimiento.

Análisis: la información fue capturada en formato electrónico y se analizó en el programa Statistics SPSS versión 22.

\section{RESULTADOS}

Se obtuvo información de 7,177 registros pertenecientes a igual número de mujeres atendidas que solicitaron ILE.

Al 100\% de las mujeres solicitantes del procedimiento se les proporcionó consejería sobre las características del procedimiento de ILE, riesgos para la salud e información sobre los métodos de anticoncepción, así como la lectura y comprensión de la carta de consentimiento informado antes de la realización de la interrupción del embarazo.

Se encontró que el grupo de edad que con mayor frecuencia se realizó la ILE fue el de 21 a 25 años, con un 34.5\% (promedio de $23.5 \pm 2.5$ años). En segundo lugar, las mujeres de 15 a 20 años, con un $26.3 \%$. Se observó que el $82.89 \%$ lo integraron las mujeres entre 15 y 30 años, lo que indica diferencias significativas con los demás grupos de edad (Chi cuadrada, $\mathrm{p}<0.001$ ). En la tabla 1 se presenta la distribución específica de cada grupo de edad según el periodo de tiempo.

El 76.45\% de las mujeres tenían un nivel de escolaridad correspondiente a secundaria y preparatoria; estos porcentajes, comparados en conjunto con los demás niveles de escolaridad (Tabla 2), mostraron diferencias estadísticamente significativas (Chi cuadrada, $\mathrm{p}<0.001)$.

En relación con el estado civil, las mujeres solteras fueron las que más solicitaron la interrupción de su embarazo, con un $54.61 \%$, seguidas por las que estaban en unión libre, con un $26.72 \%$. También se encontraron diferencias significativas al compararse estos valores con los demás subgrupos (Chi cuadrada, p < 0.001), como se observa en la tabla 3.

$\mathrm{Al} \mathrm{100 \%} \mathrm{de} \mathrm{las} \mathrm{mujeres} \mathrm{que} \mathrm{se} \mathrm{les} \mathrm{realizó}$ ILE se les ofreció y otorgó algún método de anticoncepción de acuerdo a las normas según su edad, condiciones clínicas y elección de las pacientes. Cabe señalar que para el primer año del programa la información no se registró ni contabilizó adecuadamente, 
Tabla 1. Distribución por grupos de edad de las mujeres que se sometieron a una ILE

\begin{tabular}{|l|c|c|c|c|c|c|c|c|}
\hline & \multicolumn{2}{|c|}{2012} & \multicolumn{2}{c|}{2013} & \multicolumn{2}{c|}{2014} & \multicolumn{2}{c|}{ Total } \\
\hline $\begin{array}{l}\text { Grupo de } \\
\text { edad }\end{array}$ & Frecuencia & $\%$ & Frecuencia & $\%$ & Frecuencia & $\%$ & Frecuencia & $\%$ \\
\hline$<$ de 15 años & 18 & 1.0 & 20 & 0.68 & 15 & 0.63 & 53 & 0.77 \\
\hline $15-20$ años & 494 & 27.40 & 772 & 26.36 & 600 & 25.13 & 1,866 & 26.30 \\
\hline $21-25$ años & 611 & 33.86 & 987 & 33.73 & 858 & 35.95 & 2,456 & 34.51 \\
\hline $26-30$ años & 375 & 20.80 & 611 & 20.90 & 522 & 21.85 & 1,508 & 21.18 \\
\hline $31-35$ años & 158 & 8.75 & 301 & 10.30 & 237 & 9.94 & 696 & 9.66 \\
\hline 36-40 años & 107 & 5.93 & 171 & 5.84 & 118 & 4.95 & 396 & 5.57 \\
\hline $41-45$ años & 35 & 1.94 & 60 & 2.05 & 34 & 1.42 & 129 & 1.80 \\
\hline 46 y más & 5 & 0.27 & 3 & 0.096 & 3 & 0.13 & 11 & 0.16 \\
\hline Sin datos & 1 & 0.05 & 1 & 0.032 & 0 & 0.0 & 2 & 0.05 \\
\hline Total & 1,804 & 100.0 & 2,926 & 100.0 & 2,387 & 100.0 & 7,117 & 100.0 \\
\hline
\end{tabular}

Chi cuadrada de homogeneidad: $p<0.001$.

Tabla 2. Distribución por nivel de escolaridad de las mujeres que se sometieron a una ILE

\begin{tabular}{|l|c|c|c|c|c|c|c|c|}
\hline & \multicolumn{2}{|c|}{2012} & \multicolumn{2}{c|}{2013} & \multicolumn{2}{c|}{2014} & \multicolumn{3}{c|}{ Total } \\
\hline Nivel escolar & Frecuencia & $\%$ & Frecuencia & $\%$ & Frecuencia & $\%$ & Frecuencia & $\%$ \\
\hline Ninguno & 16 & 0.89 & 16 & 0.57 & 14 & 0.59 & 46 & 0.64 \\
\hline Primaria & 114 & 6.32 & 209 & 7.15 & 145 & 6.06 & 468 & 6.57 \\
\hline Secundaria & 529 & 29.32 & 990 & 33.82 & 779 & 32.63 & 2,298 & 32.28 \\
\hline Preparatoria & 829 & 45.93 & 1,263 & 43.16 & 1,052 & 44.09 & 3,144 & 44.17 \\
\hline $\begin{array}{l}\text { Profesional } \\
\text { y posgrado }\end{array}$ & 311 & 17.24 & 448 & 15.30 & 395 & 16.55 & 1,154 & 16.21 \\
\hline Sin datos & 5 & 0.30 & 0 & 0.0 & 2 & 0.08 & 7 & 0.10 \\
\hline Total & 1,804 & 100.0 & 2,926 & 100.0 & 2,387 & 100.0 & 7,117 & 100.0 \\
\hline
\end{tabular}

Chi cuadrada de homogeneidad: $p<0.001$

por lo que las cifras para esta variable corresponden al periodo de 2013-2014.

Los métodos hormonales fueron los más frecuentes, con un $75.77 \%$ (Chi cuadrada, $\mathrm{p}<0.001$ ), en comparación con los demás métodos (Tabla 4).

Como dato complementario, se realizaron 303 vasectomías a las parejas de las mujeres que se les otorgó algún método de anticoncepción temporal; esta cifra representó el 5.7\% de las mujeres que solicitaron ILE.

\section{DISCUSIÓN}

Según la encuesta entre la juventud ENJUVE 2010, realizada en México ${ }^{5}$, el 12.6\% de las jóvenes entre 12 y 29 años afirmaron 
Tabla 3. Distribución por estado civil de las mujeres que se sometieron a una ILE

\begin{tabular}{|l|c|c|c|c|c|c|c|c|}
\hline & \multicolumn{2}{|c|}{2012} & \multicolumn{2}{c|}{2013} & \multicolumn{3}{c|}{2014} & \multicolumn{2}{c|}{ Total } \\
\hline Estado civil & Frecuencia & $\%$ & Frecuencia & $\%$ & Frecuencia & $\%$ & Frecuencia & $\%$ \\
\hline Soltera & 975 & 54.06 & 1,547 & 52.88 & 1,365 & 57.18 & 3,887 & 54.61 \\
\hline Unión libre & 472 & 26.16 & 801 & 27.37 & 629 & 26.35 & 1,902 & 26.72 \\
\hline Casada & 168 & 9.33 & 312 & 10.66 & 199 & 8.34 & 679 & 9.54 \\
\hline Separada & 148 & 8.17 & 209 & 7.15 & 150 & 6.28 & 507 & 7.12 \\
\hline Divorciada & 30 & 1.68 & 46 & 1.57 & 39 & 1.62 & 115 & 1.61 \\
\hline Viuda & 5 & 0.26 & 10 & 0.034 & 4 & 0.17 & 19 & 0.26 \\
\hline Sin datos & 6 & 0.34 & 1 & 0.032 & 1 & 0.06 & 8 & 0.20 \\
\hline Total & 1,804 & 100.0 & 2,926 & 100.0 & 2,387 & 100.0 & 7,117 & 100.0 \\
\hline
\end{tabular}

Chi cuadrada de homogeneidad: $p<0.001$.

Tabla 4. Método de anticoncepción elegido por la mujer posterior a la realización de una ILE

\begin{tabular}{|l|c|c|c|c|c|c|c|c|}
\hline & \multicolumn{2}{|c|}{2012} & \multicolumn{2}{c|}{2013} & \multicolumn{2}{c|}{$\mathbf{2 0 1 4}$} & & \\
\hline Método & $<\mathbf{2 0}$ años & $>\mathbf{2 0}$ años & $<\mathbf{2 0}$ años & $\mathbf{> 2 0}$ años & $<\mathbf{2 0}$ años & $>\mathbf{2 0}$ años & Frecuencia & $\%$ \\
\hline Hormonal $^{\dagger}$ & $*$ & $*$ & 433 & 1,759 & 324 & 1,510 & 4,026 & 75.77 \\
\hline DIU & $*$ & $*$ & 95 & 434 & 4 & 95 & 628 & 11.83 \\
\hline Preservativo & $*$ & $*$ & 72 & 133 & 148 & 306 & 659 & 12.40 \\
\hline Total & $*$ & $*$ & 600 & 2,326 & 476 & 1,911 & 5,313 & 100.0 \\
\hline Vasectomía & 0 & 92 & 0 & 138 & 0 & 73 & 303 & 100.0 \\
\hline
\end{tabular}

Chi cuadrada de homogeneidad: $p<0.001$

DIU: dispositivo intrauterino.

"Sin datos.

†Incluye implante subdérmico, parches, orales e inyectables.

haber interrumpido un embarazo, es decir, poco más de un millón de mujeres, de las cuales las de Ciudad de México ocuparon el primer lugar, con un $21.5 \%$, seguidas por las de Puebla (18.9\%), San Luis Potosí (17.6\%) y Estado de México (17.3\%). Este grupo de edad es consistente con lo encontrado en el presente estudio, en el que se observó que las mujeres que más se sometieron a un ILE tenían entre 15 y 30 años, con un $81.89 \%$.

El GIRE ${ }^{7}$ reportó el perfil general social de las usuarias que se hicieron ILE en la Ciudad de México entre 2007 y 2014, y menciona que en dicho periodo se atendieron 123,642 mujeres para realizarse una ILE. En dicho reporte se describe lo siguiente: en su mayoría las mujeres eran residentes de Ciudad de México (72\%), el nivel educativo predominante fue preparatoria (39.2\%), la mayoría eran solteras $(52 \%)$ y la principal ocupación era la de ama de casa (36\%). Además, se indica que el método más empleado para la ILE fue con medicamentos (70\%), seguido por aspiración manual endouterina (27\%) y 
legrado uterino instrumentado $(2.5 \%)^{7}$. Los resultados de nuestro trabajo indican que la mayoría tenían un nivel escolar correspondiente a secundaria y preparatoria (76\%), y en lo que respecta al estado civil, las más frecuentes fueron las solteras, con un $54 \%$, seguidas por las que estaban en unión libre, con un $26 \%$.

$\mathrm{El}$ aborto es controversial, ya que involucra aspectos relacionados con los derechos humanos, sexuales y reproductivos de las mujeres; con las leyes y las políticas en materia de salud; con los valores éticos, morales y religiosos; con condiciones socioeconómicas de las mujeres, y con las ideas que predominan en nuestra cultura ${ }^{6}$. Al respecto, la labor de consejería es muy importante, y por esta razón en nuestro trabajo al 100\% de las mujeres se les otorgó una plática de consejería para que estuvieran informadas no sólo del procedimiento del ILE, sino también de los aspectos legales, éticos, riesgos, así como de la información necesaria para la utilización de métodos anticonceptivos posteriores a la intervención.

Las anteriores acciones realizadas con las mujeres que se practicaron ILE responden a las estrategias y líneas de acción para la atención de la salud reproductiva en México a través de sus tres ejes rectores: el acceso a la información, el derecho a la libre decisión y la prestación de servicios con calidad $^{8,9}$, acciones que desde el año 2001 y hasta la fecha se impulsan, apoyan y promueven en nuestro país, y son acordes con las directrices de atención para el aborto de la Organización Mundial de la Salud (OMS) ${ }^{2}$.

En este reporte se ha presentado un panorama general de la situación del Programa de Interrupción Legal del Embarazo en una unidad de salud de primer nivel de atención en la Ciudad de México; aunque el periodo de tiempo no es representativo y es una limitante de nuestro estudio, el tamaño de la muestra es considerado como suficiente para que, sin generalizar hacia otras unidades de salud, se considere como un acercamiento de lo que ha sucedido en torno a este programa desde que se lleva a cabo de forma oficial en México.

Es de llamar la atención que, a pesar de que el Programa de Interrupción Legal del Embarazo está dirigido a las mujeres que desean de alguna forma interrumpir el embarazo, un porcentaje pequeño de sus parejas solicitaron como método de anticoncepción la vasectomía. Este hecho es considerado un hallazgo importante a considerar, ya que «con lógica y de forma tradicional» la consejería en anticoncepción para la mujer ofrece métodos propios para ella, sin considerar la vasectomía. Al respecto, Ipas ${ }^{10}$, organización no gubernamental que trabaja para ampliar el acceso a la atención del aborto y mejorar la calidad de los servicios de salud reproductiva en México, así como para promover los derechos sexuales y reproductivos de las mujeres, difunde en muchos países del mundo las diferentes ofertas de consejería en anticoncepción; sin embargo, no considera la vasectomía como una posibilidad adicional para la mujer a través de su pareja ${ }^{11}$, recomendando sólo métodos de anticoncepción postaborto exclusivos para su uso para la mujer.

En el mismo contexto, la International Planned Parenthood Federation ${ }^{12}$ publica, en sus guías clínicas y protocolos para abortos de primer trimestre, los procedimientos quirúrgicos y médicos, los lineamientos y las recomendaciones postaborto y, de igual forma, no se considera la participación del hombre por medio de la vasectomía.

Vale la pena señalar que la Agenda 2030 para el Desarrollo Sostenible (ADS) $)^{13}$ ofrece «nuevos objetivos mundiales y compromisos renovados de los gobiernos del mundo para reducir la mortalidad materna; lograr acceso universal a información, educación y servicios de salud sexual y reproductiva; garantizar los derechos reproductivos, y lograr la igualdad de género como un asunto de derechos humanos de mujeres y niñas». Al respecto, los objetivos de desarrollo dan prioridad a algunos de los mayores retos 
que enfrentan las adolescentes y mujeres jóvenes, tales como matrimonio infantil, violencia de género y falta de acceso a servicios de salud sexual y reproductiva. Este trabajo responde de alguna forma contribuyendo a las políticas de la ADS.

A nivel regional, lo descrito en este reporte contribuye a la generación de información general y útil para la planeación, diseño y orientación de las políticas de atención y mercadotecnia social en salud ${ }^{14} \mathrm{de}$ las mujeres que solicitan ILE, y se destaca la labor de consejería y el otorgamiento de métodos de anticoncepción, incluida la vasectomía.

Los autores declaramos que no existen conflictos de intereses.

\section{BIBLIOGRAFÍA}

1. Diagnóstico y tratamiento del aborto espontáneo y manejo inicial del aborto recurrente. México: Secretaría de Salud; 2009. [Citado el 15 de octubre de 2014]. Disponible en: http://www. cenetec.salud.gob.mx/descargas/gpc/CatalogoMaestro/088_ GPC_Abortoespyrecurrente/ABORTO_EVR_CENETEC.pdf

2. Organización Mundial de la Salud. Aborto sin riesgos: Guía técnica y de políticas para sistemas de salud. 2.a ed. 2012. [Citado el 10 de octubre de 2014]. Disponible en: http://apps.who.int/iris/ bitstream/10665/77079/1/9789243548432_spa.pdf

3. Centro de noticias ONU. OMS alerta sobre el aumento de abortos inseguros en países en desarrollo. [Citado el 25 de octubre de 2014]. Disponible en: http://www.un.org/spanish/News/ story.asp?NewsID=22576\#.VF5pN_mG9Pc
4. Belkis AG, Wilches GJL, Figueroa LA. Interrupción legal del embarazo; recursos disponibles en la Secretaría de Salud del Distrito Federal. 1.a ed. México: Instituto Nacional de Salud Pública; 2009. p. 15-20.

5. Noveno Encuentro Nacional Feminista 2013 Jalisco, México. La interrupción legal del embarazo: ILE. p. 2-4. [Citado el 5 de octubre de 2014]. Disponible en: http://enf2013.mx/portal/descargas/programa/Documento-Base-29-sept-final.pdf

6. González LAD, Billings DL. El aborto en México. Ipas; 2002. [Citado el 16 de octubre de 2014]. Disponible en: http://ccp.ucr. ac.cr/ac/gonzalez.pdf

7. Grupo de Información en Reproducción Elegida (GIRE). Perfil de las usuarias que han realizado interrupción legal del embarazo en la Ciudad de México. Abril 2007 a junio 2014. [Citado el 21 de noviembre de 2014]. Disponible en: http://andar.org.mx/img/ docs_pdf/ile_web_jun2014.pdf

8. Programa de acción: Salud Reproductiva. 1. ${ }^{\text {a }}$ ed. México: Secretaría de Salud; 2001. p. 9.

9. Norma Oficial Mexicana NOM-005-SSA2-1993 de los Servicios de Planificación Familiar. D.O.F. 30-V-1994. Última reforma; D.O.F. 21-I-2004.

10. Promoviendo la salud de las mujeres. Promoviendo el respeto a sus derechos reproductivos. México: Ipas. [Citado el 10 de enero de 2017]. Disponible en: http://ipasmexico.blogspot. $\mathrm{mx} / 2008 / 09 /$ qu-es-ipas.html

11. Promoviendo la salud de las mujeres. Promoviendo el respeto a sus derechos reproductivos. El inicio de la anticoncepción después de un aborto con medicamentos inducido en el primer trimestre. Ipas. [Citado el 12 de enero de 2017]. Disponible en: http://www.ipas.org/ /media/Files/lpas\%20Publications/ MAFactsheet1Sp.ashx

12. Guías clínicas y protocolos para abortos de primer trimestre. Procedimientos quirúrgicos y médicos. IPPF (International Planned Parenthood Federation). [Citado el 20 de enero de 2017]. Disponible en: https://www.ippfwhr.org/sites/default/files/Gu\%C3\%ADas\%20cl\%C3\%ADnicas\%20y\%20protocolos.pdf

13. Promoviendo la salud y los derechos sexuales y reproductivos de las adolescentes y mujeres jóvenes: enfoque en el aborto seguro en la Agenda 2030 para el Desarrollo Sostenible. [Citado el 20 de enero de 2017]. Disponible en: http://repositorio.gire.org. $\mathrm{mx} /$ bitstream/123456789/2342/1/PromoviendoDSYR.pdf

14. Manual de Mercadotecnia Social en Salud. Dirección General de Promoción de la Salud. Dirección de Estrategias y Desarrollo de Entornos Saludables. Subdirección de Mercadotecnia Social. 1.a ed. México: Secretaría de Salud; 2010. 